\title{
BMJ Open Perfusion parameters and target values during extracorporeal cardiopulmonary resuscitation: a scoping review protocol
}

\author{
Lars Saemann, ${ }^{\oplus 1,2}$ Christine Schmucker, ${ }^{3}$ Lisa Rösner, ${ }^{1}$ Friedhelm Beyersdorf, ${ }^{1}$ \\ Christoph Benk ${ }^{1}$
}

To cite: Saemann L, Schmucker C, Rösner L, et al. Perfusion parameters and target values during extracorporeal cardiopulmonary resuscitation: a scoping review protocol. BMJ Open 2019;9:e030562. doi:10.1136/ bmjopen-2019-030562

- Prepublication history for this paper is available online. To view these files, please visit the journal online (http://dx.doi. org/10.1136/bmjopen-2019030562).

Received 20 March 2019 Revised 09 August 2019 Accepted 13 August 2019

Check for updates

\section{(C) Author(s) (or their} employer(s)) 2019. Re-use permitted under CC BY-NC. No commercial re-use. See rights and permissions. Published by BMJ.

1Department of Cardiovascular Surgery, University Hospital Freiburg Cardiac Centre Freiburg Bad Krozingen Freiburg Branch, Freiburg, Germany

2Department of Cardiac Surgery, University Hospital Heidelberg, Heidelberg, Germany

${ }^{3}$ Institute for Evidence in

Medicine (for Cochrane

Germany Foundation), Medical

Center, Faculty of Medicine,

University of Freiburg, Freiburg,

Freiburg, Germany

Correspondence to

Lars Saemann;

lars.saemann@gmx.de

\section{ABSTRACT}

Introduction Extracorporeal cardiopulmonary resuscitation (eCPR) is increasingly applied in out-ofhospital cardiac arrest (OHCA) and in-hospital cardiac arrest (IHCA) patients. Treatment results are promising, but the efficacy and safety of the procedure are still unclear. Currently, there are no recommended target perfusion parameters during eCPR, the lack of which could result in inadequate (re)perfusion. We aim to perform a scoping review to explore the current literature addressing target perfusion parameters, target values, corresponding survival rates and neurologic outcomes in OHCA and IHCA patients treated with ECPR.

Methods and analysis To identify relevant research, we will conduct searches in the electronic databases MEDLINE, EMBASE, Social Science Citation Index, Social Science Citation Index Expanded and the Cochrane library. We will also check references of relevant articles and perform a cited reference research (forward citation tracking).

Two independent reviewers will screen titles and abstracts, check full texts for eligibility and perform data extraction. We will resolve dissent by consensus, moderated by a third reviewer. We will include observational and controlled studies addressing target perfusion parameters and outcomes such as survival rates and neurologic findings in $\mathrm{OHCA}$ and IHCA patients treated with eCPR. Data extraction tables will be set up, including study and patients' characteristics, aim of study, details on eCPR including target perfusion parameters and reported outcomes. We will summarise the data using tables and figures (ie, bubble plot) to present the research landscape and to describe potential clusters and/or gaps.

Ethics and dissemination An ethical approval is not needed. We intend to publish the scoping review in a peer-reviewed journal and present results on a scientific meeting.

\section{INTRODUCTION \\ Background}

Out-of-hospital cardiac arrest (OHCA) and in-hospital cardiac arrest (IHCA) are associated with poor results. ${ }^{1-6}$ In both locations of cardiac arrest (CA), survival rate with a favourable neurological outcome, expressed by a cerebral performance category score of $1-2$, is low. ${ }^{78}$ The initial strategy is to bridge

\section{Strengths and limitations of this study}

- We will perform a comprehensive systematic literature search addressing target parameters and target values, as well as survival rates and neurologic outcomes in extracorporeal cardiopulmonary resuscitation (eCPR) applied in adult out-of-hospital cardiac arrest and in-hospital cardiac arrest patients regardless of comorbidities.

- The scoping review will be reported according to the preferred reporting items for systematic review and meta-analysis statement for scoping reviews and, therefore, will be conducted in line with 'the stateof-the-art' criteria.

- Two members of the team will independently conduct the entire study inclusion (literature screening) and data extraction process, to avoid bias during research process

- In order to avoid the common heterogeneity of outcome presentation in ECPR literature, we clearly predefined outcome variables, which will be incorporated in the scoping review.

- The language of articles, which will be included, is restricted to English and German.

CA by any kind of cardiopulmonary resuscitation (CPR), so called conventional CPR, mechanically performed chest compression CPR (mCPR) or, recently, extracorporeal CPR (eCPR). eCPR requires an extracorporeal mechanical circulatory support. In this setup, perfusion of the body is restored and maintained by an extracorporeal life support (ECLS) device, also called venoarterial extracorporeal membrane oxygenation (VA-ECMO).${ }^{9}{ }^{10}$ An outflow cannula is usually placed in the femoral and/or jugular vein and the femoral artery usually serves as an access for inflow of the artificially oxygenated blood. ${ }^{11}$ In order to improve outcomes of both OHCA and IHCA, some groups started to extend their CPR programme by eCPR. Different guidelines and consensus papers on this topic have recently been published. ${ }^{712-14}$ 


\begin{tabular}{ll}
\hline Table 1 & Search items \\
\hline No & Search items (controlled terms) \\
\hline 1 & eCPR \\
2 & VA ECMO \\
3 & ECMO cardiac arrest resuscitation \\
4 & ECLS \\
\hline
\end{tabular}

ECLS, extracorporeal life support; ECMO, extracorporeal membrane oxygenation; eCPR, extracorporeal cardiopulmonary resuscitation;VA, veno arterial.

However, whether they are based on the best available evidence is currently questionable.

\section{Rationale}

Results of eCPR in OHCA and IHCA are promising but still not satisfactory. ${ }^{1516}$ The 'right' choice and adjustment of perfusion parameters as target parameters during eCPR seem to be heterogenic. ${ }^{121315-17}$ Brooks et al, as well as Grunau et al, pointed out the differing framework of eCPR in OHCA of various hospitals and also mentioned a variable description of practice and patient inclusion in eCPR in OHCA. ${ }^{17} 18$ Heterogenic implementation of perfusion practice during eCPR may lead to suboptimal reanimation results and may inhibit both efficacy and safety of eCPR as a treatment option for IHCA and OHCA.

\section{Objectives}

It is necessary to systematically review eCPR literature addressing associations between perfusion parameters and target values as well as survival rates and neurologic outcomes. Therefore, the planned scoping review will address the following questions:

1. Which perfusion parameters are used as target parameters during eCPR in IHCA and OHCA, respectively?

2. What are the target values of these targeted perfusion parameters in IHCA and OHCA, respectively?

3. What are the respective survival rates and neurological outcome scores to hospital discharge?

\section{METHODS AND ANALYSIS}

This protocol is written with reference to the preferred reporting items for systematic review and meta-analysis protocols statement ${ }^{19}$ and 'a priori' defines the following methodology on which the scoping review will be based on the following:

\section{Eligibility criteria}

We will include any controlled clinical study design (randomised controlled trials and non-randomised controlled trials) providing information on perfusion target parameters and on survival rates and neurological outcomes in adults ( $>18$ years old) treated with eCPR, with the need of resuscitation of presumed cardiac origin. We will exclude patients who received any kind of mechanical circulatory support immediately after cardiac surgery due to failed weaning from cardiopulmonary bypass. Case studies and articles which do not clearly distinguish between OHCA and IHCA will also be excluded. CA of pulmorespiratory origin is an exclusion criterium, as well.

\section{Information sources}

Our search terms will combine controlled terms and free-text searches (table 1). The search strategies will be adapted to each database. We will develop the final search strategy in collaboration with an expert medical sciences librarian. To identify relevant research, we will conduct searches from the beginning of eCPR in the electronic databases MEDLINE via PubMed, EMBASE, Social Science Citation Index via Web of Science, Social Science Citation Index Expanded and the Cochrane library. We will also check references of relevant articles and perform a cited reference research (forward citation tracking). The language of articles, which will be included, is restricted to English and German. Owing to the research question, we decided to conduct a broad search, that is, including more specific search terms would, in our case, be associated with a higher risk of missing wrongly indexed studies in the literature.

Table 2 Study key characteristics

\begin{tabular}{lll}
\hline Type of information & No & Attribute \\
\hline $\begin{array}{l}\text { Study characteristics } \\
\text { Patient characteristics }\end{array}$ & 1 & (a) Country of the study, (b) sample size \\
\hline Intervention characteristics & 2 & $\begin{array}{l}\text { Place of CA: OH/IH } \\
\text { (a) Start of eCPR: OH/IH }\end{array}$ \\
& $\begin{array}{l}\text { (b) All perfusion parameters which are used as target parameters during eCPR } \\
\text { (Question 1) }\end{array}$ \\
$\begin{array}{l}\text { Information on subsequent exclusion } \\
\text { of the study based on intervention } \\
\text { characteristics }\end{array}$ & $\begin{array}{l}\text { (a) Contemption of eligibility criteria } \\
\text { (b) Target parameters given but target values missing or target values }\end{array}$ \\
& $\begin{array}{l}\text { erroneously given as calculated means of the entire population } \\
\text { (c) No information given on perfusion parameters in terms of target parameters }\end{array}$
\end{tabular}

CA, cardiac arrest;eCPR, extracorporeal cardiopulmonary resuscitation; IH, in-hospital; $\mathrm{OH}$, out-of-hospital. 
Table 3 Outcome variables

\begin{tabular}{lll}
\hline Type of information & No & Attribute \\
\hline Outcome variable & 1 & (a) Survival rate to hospital discharge (Question 3) \\
$\begin{array}{ll}\text { Information on subsequent exclusion of } \\
\text { the study based on outcome variables }\end{array}$ & $\begin{array}{l}\text { (b) Neurological outcome to hospital discharge (Question 3) } \\
\text { (b) No information given on time frame of survival rate or neurologic outcome, } \\
\text { or time frame incomparable to majority of other studies }\end{array}$
\end{tabular}

For database, MEDLINE literature search strategy will be as follows: (eCPR) OR ('VA ECMO') OR ('ECMO cardiac arrest resuscitation') OR (ECLS).

\section{Review process}

Two independent reviewers will screen titles and abstracts, check full texts for eligibility and perform data extraction. We will resolve dissent by consensus moderated by a third reviewer. As known for scoping reviews, the methodology may be adapted minimally during the review process itself in terms of eligibility criteria, study characteristics and outcome variables. ${ }^{20}{ }^{21}$ As mentioned in popular literature, which describes the methodology of scoping reviews, risk of bias assessment will not be part of the scoping review process. ${ }^{22} 23$

\section{Data items}

Data extraction tables will be set up in MS Excel including study (eg, first author, DOI) and patients' characteristics (eg, age, CPR technique, comorbidities, OHCA and IHCA), aim of study, details on eCPR including target perfusion parameters and reported outcomes (table 2). Variables in section No 3 may be extended during the review process, for example, number of organ donation after unsuccessful eCPR, as Ortega-Deballon $e t$ al stated this as an important side topic of reanimation. ${ }^{24}$

\section{Outcomes}

Outcome variables are listed in table 3 .

\section{Data synthesis}

We expect dramatically heterogeneous study characteristics within the field of eCPR. This may express itself both in patient population and eCPR procedure as well as eCPR experience. Therefore, as a first step of our eCPR literature research project, we will summarise the data using tables and figures (ie, bubble plot) to present the research landscape and to describe potential clusters and/or gaps.

Results will be presented in two tables (see above). One table serves for IHCA and one serves for OHCA. In case of an OHCA, eCPR can either be started $\mathrm{OH}$, or IH, if the patient is transported to the hospital under mCPR. Some groups might also change the eCPR regimen after arrival in the hospital. In consequence, the table which presents data for OHCA will be separated into two major columns, which refer to the location of eCPR start. In both tables, studies will be listed chronologically, beginning with the latest publication first. In addition to the tabulated presentation of data, each target parameter will be addressed and discussed in a separate text.

\section{Patient and public involvement}

Patients or public will not be involved.

\section{Perspective}

If the scoping review provides us with enough study data, we plan a meta-analysis in a second research project to compare efficacy and safety of eCPR in patients with IHCA and OHCA.

\section{DISSEMINATION}

We intend to publish the review in a peer-reviewed journal. Besides, we may also present the results on a scientific conference.

Contributors LS is the guarantor and drafted most of the protocol. CS also drafted parts of the protocol and gave substantial information and support for good practice in systematic reviewing. LR supported drafting process. FB and CB contributed as experts forextracorporeal cardiopulmonary resuscitation and provided scientific knowledge. The purpose of developing this review was initiated by CB. All authors read and provided feedback on the final version of the protocol.

Funding The authors have not declared a specific grant for this research from any funding agency in the public, commercial or not-for-profit sectors.

Competing interests $\mathrm{FB}$ und $\mathrm{CB}$ are shareholder of Resuscitec $\mathrm{GmbH}$.

Patient consent for publication Not required.

Ethics approval This study uses only data that have already been published and does not need any ethical approval.

Provenance and peer review Not commissioned; externally peer reviewed.

Open access This is an open access article distributed in accordance with the Creative Commons Attribution Non Commercial (CC BY-NC 4.0) license, which permits others to distribute, remix, adapt, build upon this work non-commercially, and license their derivative works on different terms, provided the original work is properly cited, appropriate credit is given, any changes made indicated, and the use is non-commercial. See: http://creativecommons.org/licenses/by-nc/4.0/.

\section{REFERENCES}

1. Cheskes S, Schmicker RH, Rea T, et al. The association between AHA CPR quality guideline compliance and clinical outcomes from out-of-hospital cardiac arrest. Resuscitation 2017;116:39-45.

2. Hawkes $\mathrm{C}$, Booth $\mathrm{S}$, Ji C, et al. Epidemiology and outcomes from out-of-hospital cardiac arrests in England. Resuscitation 2017;110:133-40. 
3. Kragholm K, Wissenberg M, Mortensen RN, et al. Bystander efforts and 1-year outcomes in out-of-hospital cardiac arrest. N Engl J Med Overseas Ed 2017;376:1737-47.

4. Murakami Y, Iwami T, Kitamura T, et al. Outcomes of Out-of-Hospital cardiac arrest by public location in the Public-Access defibrillation era. J Am Heart Assoc 2014;3.

5. Nolan JP, Soar J, Smith GB, et al. Incidence and outcome of inhospital cardiac arrest in the United Kingdom national cardiac arrest audit. Resuscitation 2014;85:987-92.

6. Schwartz BC, Jayaraman D, Warshawsky PJ. Survival from inhospital cardiac arrest on the internal medicine clinical teaching unit. Can J Cardiol 2013;29:117-21.

7. Link MS, Berkow LC, Kudenchuk PJ, et al. Part 7: adult advanced cardiovascular life support: 2015 American heart association guidelines update for cardiopulmonary resuscitation and emergency cardiovascular care. Circulation 2015;132(18 Suppl 2):444-64.

8. Karam N, Marijon E, Dumas F, et al. Characteristics and outcomes of out-of-hospital sudden cardiac arrest according to the time of occurrence. Resuscitation 2017;116:16-21.

9. Abrams D, Brodie D. Extracorporeal Membrane Oxygenation for Adult Respiratory Failure. Chest 2017;152:639-49.

10. Napp LC, Kühn C, Bauersachs J. Ecmo bei Herz-Kreislauf-Stillstand und kardiogenem Schock. Herz 2017;42:27-44.

11. Raffa GM, Kowalewski M, Brodie D, et al. Meta-Analysis of peripheral or central extracorporeal membrane oxygenation in Postcardiotomy and Non-Postcardiotomy shock. Ann Thorac Surg 2019;107:311-21.

12. Ellouze $\mathrm{O}$, Vuillet $\mathrm{M}$, Perrot J, et al. Comparable outcome of out-ofhospital cardiac arrest and in-hospital cardiac arrest treated with extracorporeal life support. Artif Organs 2018;42:15-21.

13. Maekawa K, Tanno K, Hase M, et al. Extracorporeal cardiopulmonary resuscitation for patients with out-of-hospital cardiac arrest of cardiac origin: a propensity-matched study and predictor analysis. Crit Care Med 2013;41:1186-96.
14. Michels G, Wengenmayer T, Hagl C, et al. Recommendations for extracorporeal cardiopulmonary resuscitation (eCPR): consensus statement of DGIIN, DGK, DGTHG, DGfK, DGNI, DGAI, DIVI and grc. Clin Res Cardiol 2018.

15. Schopka S, Philipp A, Lunz D, et al. Single-Center experience with extracorporeal life support in 103 nonpostcardiotomy patients. Artif Organs 2013;37:150-6.

16. Rousse N, Robin E, Juthier F, et al. Extracorporeal life support in outof-hospital refractory cardiac arrest. Artif Organs 2016;40:904-9.

17. Brooks SC, Shemie SD, Torrance S, et al. Barriers and opportunities related to extracorporeal cardiopulmonary resuscitation for out-ofhospital cardiac arrest in Canada: a report from the first meeting of the Canadian ECPR research Working group. CJEM 2018;20:507-17.

18. Grunau B, Hornby L, Singal RK, et al. Extracorporeal cardiopulmonary resuscitation for refractory out-of-hospital cardiac arrest: the state of the evidence and framework for application. Can $J$ Cardiol 2018;34:146-55.

19. Moher D, Shamseer L, Clarke M, et al. Preferred reporting items for systematic review and meta-analysis protocols (PRISMA-P) 2015 statement. Syst Rev 2015;4:1.

20. Levac D, Colquhoun H, O'Brien KK. Scoping studies: advancing the methodology. Implementation Sci 2010;5

21. Arksey H, O'Malley L. Scoping studies: towards a methodological framework. Int J Soc Res Methodol 2005;8:19-32.

22. Grant MJ, Booth A. A typology of reviews: an analysis of 14 review types and associated methodologies. Health Info Libr J 2009;26:91-108.

23. Higgins JPT, Green S. Cochrane Handbook for Systematic Reviews of Interventions: Version 5.1.0, 2011. Available: https://training. cochrane.org/handbook

24. Ortega-Deballon I, Hornby L, Shemie SD, et al. Extracorporeal resuscitation for refractory out-of-hospital cardiac arrest in adults: a systematic review of international practices and outcomes. Resuscitation 2016;101:12-20. 